\title{
Strong amplitude-phase coupling in submonolayer quantum dots
}

Cite as: Appl. Phys. Lett. 109, 201102 (2016); https://doi.org/10.1063/1.4967833

Submitted: 20 July 2016 . Accepted: 02 November 2016 . Published Online: 14 November 2016

Bastian Herzog, Benjamin Lingnau, Mirco Kolarczik, Yücel Kaptan, Dieter Bimberg, André Maaßdorf, Udo W. Pohl, Ricardo Rosales (D), Jan-Hindrik Schulze, Andre Strittmatter (iD, Markus Weyers, Ulrike Woggon, Kathy Lüdge, and Nina Owschimikow

ARTICLES YOU MAY BE INTERESTED IN

Dynamic phase response and amplitude-phase coupling of self-assembled semiconductor quantum dots

Applied Physics Letters 110, 241102 (2017); https://doi.org/10.1063/1.4985705

Fast gain and phase recovery of semiconductor optical amplifiers based on submonolayer quantum dots

Applied Physics Letters 107, 201102 (2015); https://doi.org/10.1063/1.4935792

Multi-stack InAs/InGaAs sub-monolayer quantum dots infrared photodetectors

Applied Physics Letters 102, 011131 (2013); https://doi.org/10.1063/1.4774383

\section{Lock-in Amplifiers up to $600 \mathrm{MHz}$}
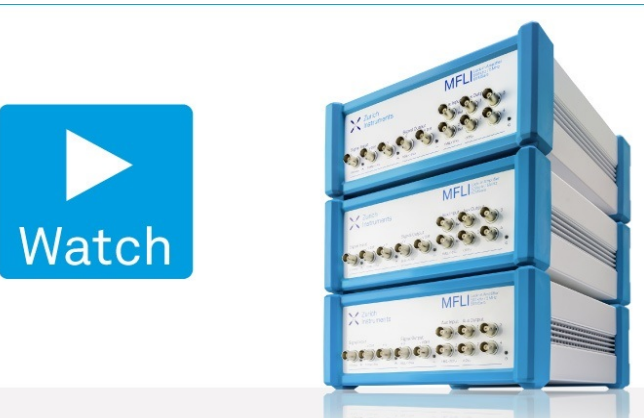


\title{
Strong amplitude-phase coupling in submonolayer quantum dots
}

\author{
Bastian Herzog, ${ }^{1, a)}$ Benjamin Lingnau, ${ }^{2}$ Mirco Kolarczik, ${ }^{1}$ Yücel Kaptan, ${ }^{1}$ Dieter Bimberg, ${ }^{3}$ \\ André Maaßdorf, ${ }^{4}$ Udo W. Pohl, ${ }^{3}$ Ricardo Rosales, ${ }^{3}$ Jan-Hindrik Schulze, ${ }^{3}$ \\ Andre Strittmatter, ${ }^{3}$ Markus Weyers, ${ }^{4}$ Ulrike Woggon, ${ }^{1}$ Kathy Lüdge ${ }^{2}$ \\ and Nina Owschimikow ${ }^{1}$ \\ ${ }^{1}$ Institut für Optik und Atomare Physik, TU Berlin, Straße des 17. Juni 135, 10623 Berlin, Germany \\ ${ }^{2}$ Institut für Theoretische Physik, TU Berlin, Hardenbergstrasse 36, 10623 Berlin, Germany \\ ${ }^{3}$ Institut für Festkörperphysik, TU Berlin, Hardenbergstrasse 36, 10623 Berlin, Germany \\ ${ }^{4}$ Ferdinand-Braun-Institut, Leibniz-Institut für Höchstfrequenztechnik, Gustav-Kirchhoff-Strasse 4, \\ 12489 Berlin, Germany
}

(Received 20 July 2016; accepted 2 November 2016; published online 14 November 2016)

\begin{abstract}
Submonolayer quantum dots promise to combine the beneficial features of zero- and two-dimensional carrier confinement. To explore their potential with respect to all-optical signal processing, we investigate the amplitude-phase coupling ( $\alpha$-parameter) in semiconductor optical amplifiers based on InAs/GaAs submonolayer quantum dots in ultrafast pump-probe experiments. Lateral coupling provides an efficient carrier reservoir and gives rise to a large $\alpha$-parameter. Combined with a high modal gain and an ultrafast gain recovery, this makes the submonolayer quantum dots an attractive gain medium for nonlinear optical signal processing. Published by AIP Publishing.
\end{abstract}

[http://dx.doi.org/10.1063/1.4967833]

Semiconductor optical amplifiers (SOAs) and lasers based on quantum-confined gain media constitute important building blocks for high speed data transmission and processing in advanced telecommunication networks. The active region consists mostly of either two-dimensional (2D) quantum wells (QWs) or an ensemble of zero-dimensional (0D) quantum dots (QDs). The former benefit from a large optical confinement factor and consequently a high modal gain, while the latter display a large material gain, faster gain recovery, and superior thermal stability. ${ }^{1-4}$ To combine the advantages of $\mathrm{OD}$ confinement with a larger areal density of localization centers, the growth of submonolayer (SML) QDs has been developed ${ }^{5}$ and demonstrated for InAs/GaAs laser structures. ${ }^{6}$ SML QDs consist of densely stacked vertically correlated InAs islands separated by few-monolayerthick GaAs spacers and have a relatively high areal density of $10^{12} \mathrm{~cm}^{-2} \cdot 7,8$ SML QDs show a combination of OD and 2D spectroscopic features, high optical gain of $90 \mathrm{~cm}^{-1}$, and fast gain and phase recovery. ${ }^{9-13}$

In contrast to atom-like gain media, in semiconductor opto-electronic devices a change in the optical gain is always accompanied by a change in the refractive index for the optical wave in the device, usually leading to a modification and chirp of a propagating optical pulse. ${ }^{14}$ The amplitude-phase coupling is caused by a coupling of the active states to the charge carrier reservoir storing the electrically injected carriers. ${ }^{15-17}$ As a measure of the amplitude-phase coupling, Henry's $\alpha$-parameter has been introduced, to quantify the broadening of the emission linewidth of a semiconductor laser. ${ }^{15}$ It relates the changes in the real and imaginary parts of the refractive index to the changing number density $N$ of carriers in the active region via $\alpha=-(4 \pi / \lambda)\left(\mathrm{d} n^{\prime} / \mathrm{d} N\right) /(\mathrm{d} g / \mathrm{d} N)$. Here, $\lambda$ is the central wavelength, $n^{\prime}$ is the real part of the refractive index, and $g$ is the

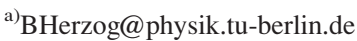

effective modal gain. Typical values for bulk and QW semiconductor lasers range from 3 to $10,{ }^{18-21}$ though a careful selection of the spectral position of the laser line in singlemode lasers allows one to push the $\alpha$-parameter to lower values. ${ }^{22}$ Generally, smaller $\alpha$-parameters are predicted for devices based on QDs. ${ }^{23-25}$ There the interdependence of gain and refractive index is reduced by decoupling the active states and the carrier reservoir, with the isolated confined states of the QDs behaving like an atomic gain medium with ideally zero linewidth enhancement. Experimentally, this has been confirmed for self-assembled QDs grown by the StranskiKrastanov (SK) method at low injection current. ${ }^{24-26}$ While for linear amplification and modulation schemes a low phase response is desirable, the opposite is true for nonlinear applications like wavelength conversion or cross phase modulation.

To assess the potential of SML QD-based opto-electronic devices in optical telecommunications and signal processing, we investigate comparatively the amplitudephase coupling in semiconductor optical amplifiers (SOAs) based on SML QDs, QWs, and SK QDs in ultrafast pumpprobe experiments and analyze the results using a rate equation model describing the carrier dynamics in the active regions. ${ }^{12}$ All studied SOAs were grown by metal organic vapor phase epitaxy according to established growth protocols. For the SML QD-based devices, the active region contains five stacks of a sixfold deposition of nominally $0.4 \mathrm{ML}$ InAs and 1.6 ML GaAs each, separated by $10 \mathrm{~nm}$ GaAs spacers. The total density of localization centers for a similar structure was about $10^{12} \mathrm{~cm}^{-2}$. 7,12 The reference device based on SK QDs is a dot-in-a-well (DWELL) structure with 7 layers of nominally $1 \mathrm{~nm} \mathrm{In}_{0,6} \mathrm{Ga}_{0.4}$ As self-assembled SK QDs, $6 \mathrm{~nm}$ capping, and $14 \mathrm{~nm}$ spacer. The capping layer acts as a 2D carrier reservoir. ${ }^{27}$ Typical QD densities in the SK growth mode are about $2 \times 10^{10}-5 \times 10^{10} \mathrm{~cm}^{-1}$. ${ }^{28}$ The QW-based reference device contains two layers of $7 \mathrm{~nm}$ 
$\mathrm{In}_{0,26} \mathrm{Ga}_{0.74}$ As with $5 \mathrm{~nm}$ GaAsP spacers. All devices contain a shallow-etched single-mode waveguide with lengths between $0.5 \mathrm{~mm}$ and $4 \mathrm{~mm}$.

Electroluminescence (EL) spectra of all three types of SOAs are shown in Figs. 1(a)-1(c) at different transparencycurrent densities $J_{\text {tr. }}$. The SK QDs have a confined ground state (GS) emitting at $1100 \mathrm{~nm}(1.13 \mathrm{eV})$ and an excited state (ES) emitting at $1060 \mathrm{~nm}(1.17 \mathrm{eV})$. The GS of the embedding QW emits at $1010 \mathrm{~nm}(1.23 \mathrm{eV})$. The broad emission originates from overlapping Gaussian-distributed confined GS and ES states. The blue-shift at higher injection currents is caused by GS saturation and increased filling of the ES of the QDs. In the case of the QW, the relatively narrow emission is defined by the low-energy edge of the QW at $1060 \mathrm{~nm}(1.17 \mathrm{eV})$ for low currents, and shows a blue-shift due to state filling in the $2 \mathrm{D}$ continuum at higher injection. For the SML QDs, the emission is narrow and centered at $965 \mathrm{~nm}(1.28 \mathrm{eV})$. The dip around $1.35 \mathrm{eV}$ is due to selfabsorption in the waveguide and disappears at high currents. The spectrum remains remarkably stable with increased injection current due to a cutoff in the exciton density of states (DOS). ${ }^{12}$

We measure changes of gain and refractive index in an ultrafast pump-probe experiment with heterodyne detection. Pump and probe pulses are derived from two simultaneously pumped highly nonlinear fibers of a Toptica FemtoFiber pro SCIR laser system. As both pulses propagate co-linearly and co-polarized in the SOA waveguide, the discrimination of pump and probe pulses is achieved in a balanced heterodyne detection scheme, where the frequency-shifted probe pulse interferes with a reference pulse. Details of the experimental approach are published in Ref. 29. This approach allows us to derive both amplitude and phase changes by comparing the complex lock-in signal $\tilde{S}(t)$ disturbed by a pump pulse to the undisturbed case $\tilde{S}_{0}(t)$ according to $\tilde{S}(t) / \tilde{S}_{0}=(|S(t)| /$ $\left.\left|S_{0}\right|\right) \exp (i \Delta \Phi(t))$, with $t$ being the pump-probe delay time. Taking the logarithm yields the differential intensity gain $\Delta G(t)=20 \log \left(|S(t)| /\left|S_{0}\right|\right)$ and the differential phase change $\Delta \Phi(t)$ of the transmitted pulse with respect to the undisturbed case.

The hatched area in Fig. 1 highlights the spectral window for the one color pump-probe experiments. Pump and probe pulses were shaped to a temporal and spectral fullwidth-at-half-maximum (FWHM) of 200 fs and $10 \mathrm{~nm}$, respectively. The pulse powers were kept at $30 \mu \mathrm{W}(0.4 \mathrm{pJ} /$ pulse) for the probe and $300 \mu \mathrm{W}(4 \mathrm{pJ} /$ pulse) for the pump pulse, which is in the linear amplification regime. Representative gain and phase recovery traces measured for the three types of SOAs are displayed in Figs. 2(a)-2(f) on a logarithmic time scale. The injection currents were between $0.8 J_{\text {tr }}$ and $3.3 J_{\text {tr }}$, where $J_{\text {tr }}$ is the respective transparency current in the investigated spectral range. The transparency current is determined from the pump-probe traces as the current at which the measured $\Delta G$ trace changes its sign from positive to negative. ${ }^{11}$ The particular values are $23 \mathrm{~mA}, 12 \mathrm{~mA}$, and $60 \mathrm{~mA}$ for the SK QDs, SML QDs, and QWs, respectively.

The gain and phase responses of the three different structures in Figs. 2(a)-2(f) show different degrees of complexity. In particular, the recovery dynamics of the SK QDs (Figs. 2(a) and 2(d)) shows features on multiple timescales. For the SML QDs, the response can be decomposed into a fast part and a slow part with characteristic times of about $2 \mathrm{ps}$ and $100 \mathrm{ps}$, respectively (Figs. 2(b) and 2(c)). ${ }^{11}$ The dynamics of the QW (apart from a spectral hole-burning process at times shorter than $1 \mathrm{ps}$ ) are reasonably well described by single-exponential fits (solid lines in Figs. 2(c) and 2(f)), leading to time constants decreasing from $330 \mathrm{ps}$ at $0.8 J_{\text {tr }}$ to $190 \mathrm{ps}$ at $3.3 J_{\text {tr }}$.

The theoretical description of the SK and SML QDs is based on rate equations for the charge-carrier dynamics of the QDs and the reservoir states, which are discussed in more detail in Refs. 12 and 30. In both structures, we classify the QD states into different subgroups, labeled by an index $j$, to account for the spectrally broad density of states. The density of states (DOS) of the SK QDs is assumed to be composed of inhomogeneously broadened ground and excited state transitions, whereas the SML QD DOS is modeled as one inhomogeneously broadened, localized DOS. The resulting rate equation system is written as

$$
\begin{gathered}
\frac{\partial}{\partial t} N^{\mathrm{res}}=J-\frac{N^{\mathrm{res}}}{\tau_{\mathrm{res}}}+\left.\frac{\partial N^{\mathrm{res}}}{\partial t}\right|_{s c} ^{c a p}, \\
\frac{\partial}{\partial t} \rho^{j}=-\frac{\left(\rho^{j}\right)^{2}}{\tau_{j}}+R_{\text {stim }}^{j}+\left.\frac{\partial \rho^{j}}{\partial t}\right|_{s c} ^{c a p}+\left.\frac{\partial \rho^{j}}{\partial t}\right|_{s c} ^{r e l},
\end{gathered}
$$

describing the carrier density in the reservoir $N^{\text {res }}$ and the occupation probabilities $\rho^{j}$, with the pump current density $J$

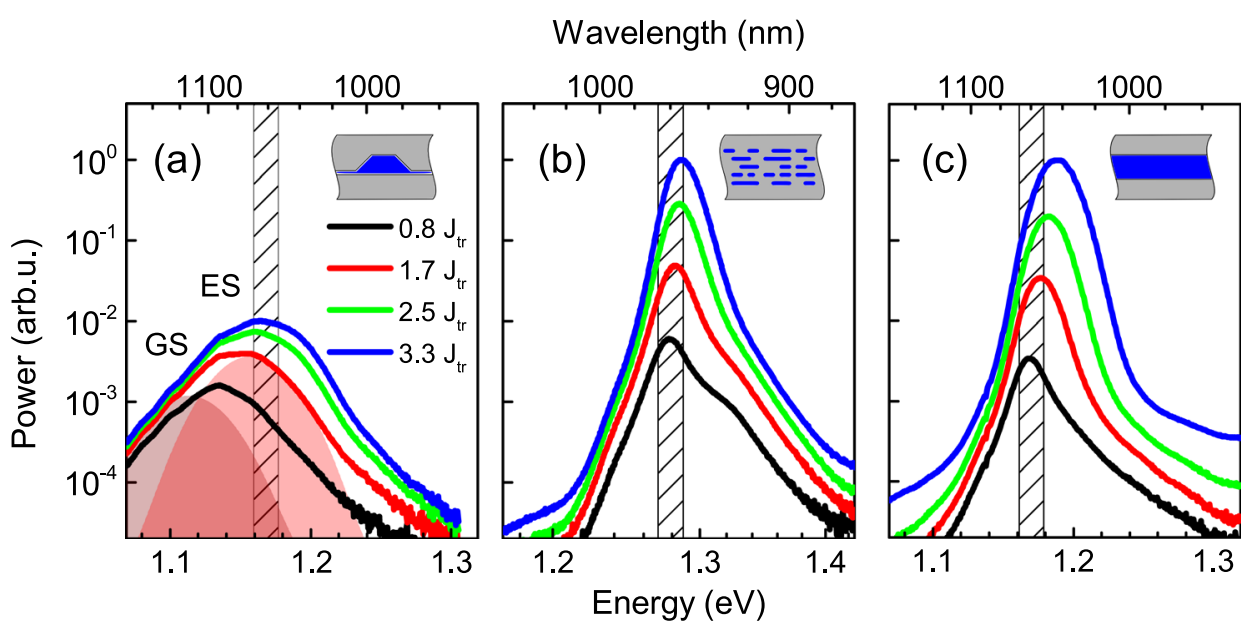

FIG. 1. Room-temperature electroluminescence spectra of SOAs based on (a) Stranski-Krastanov quantum dots, (b) submonolayer quantum dots, and (c) quantum wells as active medium. The hatched areas mark the spectral ranges of the one color pump probe experiments, and $J_{t r}$ is the respective transparency current. The insets show schematics of the respective confinement structures. The red filled area in (a) shows the deconvoluted spectra at $J=1.7 J_{t r}$ including the Gaussian distributed ground state (GS) and excited state (ES). 

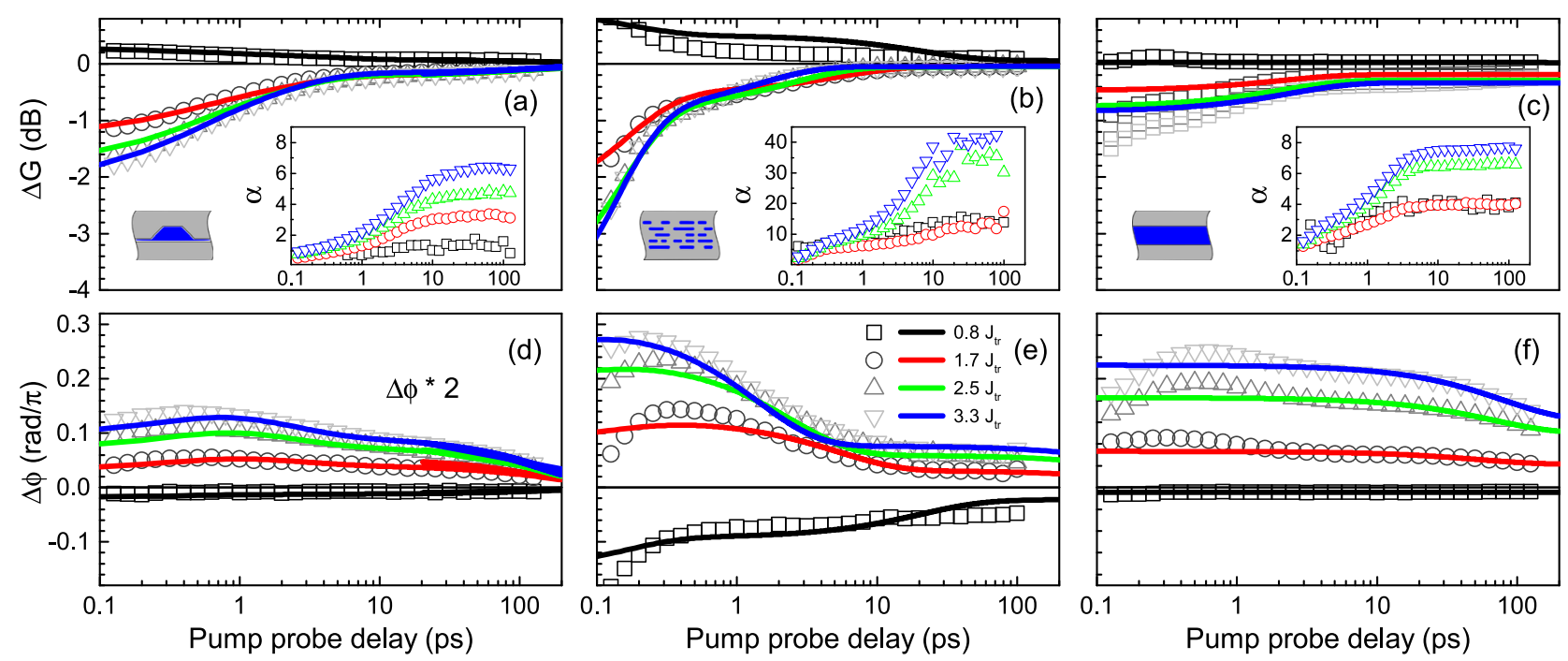

FIG. 2. (a)-(c) Gain and (d)-(f) phase dynamics of SK QD, SML QD, and QW-based devices at different injection currents. Open symbols mark the experimental data, the results of numerical simulations [(a), (b), (d), and (e)] and a single-exponential fit [(c) and (f)] are represented by solid lines. The insets show the respective time-dependent $\alpha$-parameters.

and the carrier lifetimes $\tau_{j}$ and $\tau_{\text {res }}$, respectively. $R_{\text {stim }}^{j}$ describes the stimulated recombination of the active states and $\left.\frac{\partial}{\partial t}\right|_{s c}$ denotes carrier-scattering contributions. The QD states are filled via direct capture processes from the reservoir,

$$
\left.\frac{\partial \rho^{j}}{\partial t}\right|_{s c} ^{c a p}=R^{\operatorname{cap}}\left[F\left(\hbar \omega^{j}, \mu, T\right)-\rho^{j}\right],
$$

which drives the QD carrier distribution towards the quasiFermi function $F$, with $\mu$ the reservoir quasi-Fermi level, and the capture rate $R^{\text {cap }}$.

In the SK QDs, we consider capture processes from the 2D reservoir and intra-dot relaxation from the ES to the GS

$$
\left.\frac{\partial \rho_{\mathrm{GS}}^{j}}{\partial t}\right|_{s c} ^{r e l}=R_{\mathrm{QD}}^{\mathrm{rel}}\left(1-\rho_{\mathrm{GS}}^{j}\right) \rho_{\mathrm{ES}}^{j}-\left.\frac{\partial \rho_{\mathrm{GS}}^{j}}{\partial t}\right|_{s c} ^{r e l, \text { out }},
$$

with a relaxation rate $R_{\mathrm{QD}}^{\mathrm{rel}}$. The corresponding escape process $\left.\frac{\partial \rho_{G S}^{j}}{\partial t}\right|_{s c} ^{r e l, o u t}$ is calculated from the detailed balance condition, ${ }^{30}$ and the corresponding reverse processes are given for the ES.

In the SML QD case, the reservoir is formed by the 3D bulk material, which leads to a strongly current-dependent capture rate. We additionally implement a diffusive relaxation between the states

$$
\left.\frac{\partial \rho_{\mathrm{SML}}^{j}}{\partial t}\right|_{s c} ^{r e l}=R_{\mathrm{SML}}^{\mathrm{rel}} \sum_{k \neq j}\left(1-\rho_{\mathrm{SML}}^{j}\right) \rho_{\mathrm{SML}}^{k}-\left.\frac{\partial \rho_{\mathrm{SML}}^{j}}{\partial t}\right|_{s c} ^{r e l, o u t},
$$

where the index $k$ runs over all SML QD subgroups. The inactive SML QD subgroup $j_{\text {inact }}$ at an energy of $1.35 \mathrm{eV}$ accounts for more than half of the total DOS and is required to describe the ultrafast gain recovery of the SML QDs but does not contribute to the stimulated emission. ${ }^{12}$

The rate-equation model with the assumptions detailed above provides a quantitative description of the gain and phase recovery we observe in our experiments (Table I). The main difference between SK QDs and SML QDs is the lateral coupling and the size of the inactive reservoir for the
TABLE I. Parameters as used in the simulations.

\begin{tabular}{lcccc}
\hline \hline \multicolumn{2}{c}{ SK QD } & & \multicolumn{2}{c}{ SML QD } \\
\cline { 5 - 5 } Symbol & Value & & Symbol & Value \\
\hline$\tau_{\text {res }}$ & $0.15 \mathrm{~ns}$ & & $\tau_{\text {res }}$ & $2 \mathrm{~ns}$ \\
$\tau_{\mathrm{GS}}$ & $0.3 \mathrm{~ns}$ & & $\tau_{\mathrm{SML}}$ & $0.2 \mathrm{~ns}$ \\
$\tau_{\mathrm{ES}}$ & $0.5 \mathrm{~ns}$ & & $60 \mathrm{~ns}^{-1} \times\left(\frac{J}{J_{t r}}\right)^{2}$ \\
$R^{\text {cap }}$ & $900 \mathrm{~ns}^{-1}$ & & $R^{\text {cap }}$ & $15 \mathrm{ps}^{\text {rel }}$ \\
$R^{\text {rel }}$ & $2.2 \mathrm{ps}^{-1}$ & $R^{\text {rel }}$ & \\
\hline \hline
\end{tabular}

latter structure, causing a phase response much larger than for the SK QDs, which are well decoupled from their carrier reservoir.

From the $\Delta G(t)$ and $\Delta \Phi(t)$ traces, we are able to extract a time-dependent $\alpha$-parameter, i.e., a snapshot of the interplay of amplitude and phase at various times after the optical perturbation. Using that $\Delta G \propto g L$, with $L$ being the device length, the $\alpha$-parameter is expressed as ${ }^{31}$

$$
\alpha^{\exp }(t)=-8.6859 \frac{\Delta \Phi(t)}{\Delta G(t)},
$$

if the phase change $\Delta \Phi$ is measured in rad, and the change of the differential intensity gain $\Delta G$ in decibel (dB). In this fashion, the $\alpha$-parameter is evaluated at every time step of the pump-probe delay.

In Fig. 2, we show the temporal development of the $\alpha$-parameters of all three devices as insets in the upper panels. Qualitatively, all curves show a similar behavior, rising from an initial value between unity for SK QDs and 2.5 for the QW within a time characteristic for the particular structure to an equilibrium value. The technologically relevant $\alpha$-parameter corresponds to this saturation value of the curves at longer delay times. The initial value of $\alpha(t)$ represents the situation in which the optically induced perturbation has not yet spread to reservoir states. The time interval within which $\alpha(t)$ reaches its equilibrium value corresponds to the characteristic time within which scattering processes 
transmit the initial perturbation to the carrier reservoir. This may involve spatial as well as energetic diffusion processes.

Both QW- and SK-QD-based devices are typical for their class in their behavior. The $\alpha$-parameter heavily depends on the injection current applied and shows large values as soon as significant density perturbations in the reservoir states are induced. ${ }^{32,33}$ The QW-based device shows the fastest saturation, as this process is mainly influenced by a rearrangement of the carriers in space. ${ }^{34}$ Independently of the delay time, the $\alpha$-parameter is consistently higher than for the SK-QD-based structure. For the SK QD device, the initial value of $\alpha$ is close to unity, and the equilibrium value is reached on a timescale of $10 \mathrm{ps}$, which reflects the fast capture rate from the reservoir. With increasing injection current, the values approach those observed for the QW. The SML QDs show a time dependence similar to the SK QD, but the magnitude of the $\alpha$-parameter is consistently higher than for both other structures. Both observations can be accounted for by the large abundance of carriers in inactive states (e.g., free carriers), which are spectrally close to the probe wavelength and give rise to optical intraband transitions.

The dependence of the equilibrium value of $\alpha_{\mathrm{eq}}$ on the injection current is plotted in Fig. 3 for all three structures. To illustrate the reproducibility of the values obtained for the SML QD-based structures, we show values for altogether four different devices taken from different parts of the wafer. The QW SOA exhibits the weakest current dependence of all systems with values increasing from 2.5 to 8 . In the case of the SK QDs, the initial value for the $\alpha$-parameter is below unity at low current and remains low below the transparency current. After that, a moderate increase follows, and at higher injection currents, the $\alpha$-parameter becomes comparable with that observed in the QW SOA. The SML QDs show the highest absolute values of the $\alpha$-parameter. Being still comparable to the QW at low injection current, the values increase dramatically to above 40 at higher current. A comparison with the rate-equation simulations shows that the ratio of active to inactive states at energies close to the probe wavelength is giving rise to this behavior. We assume that the hetero-confined charge carriers in SML QDs ${ }^{13}$ lead to a strong refractive index modulation due to a background of unbound electrons. As a mixed dimensional system, an

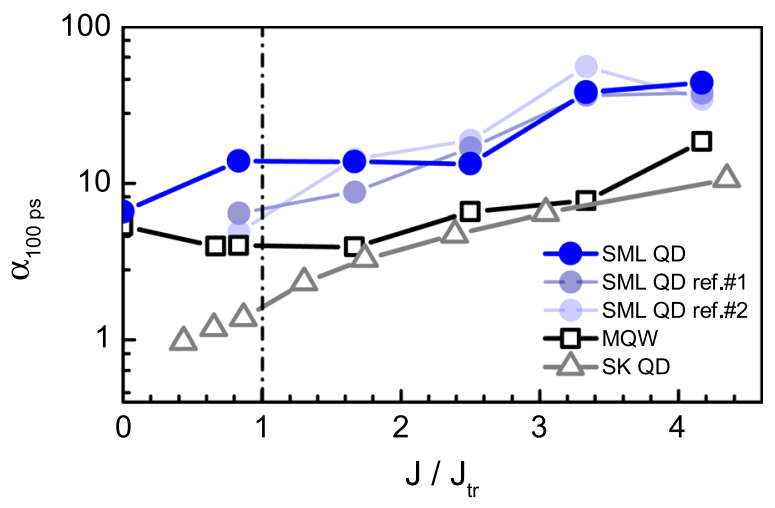

FIG. 3. Quasi-equilibrium value of $\alpha$ at 100 ps after pump-probe overlap versus injection current for all three systems. The three blue curves for the SML QDs are results for devices processed from different points on the wafer. enlargement of the SML dot sizes would increase the electron binding energy leading to OD confinement, while a reduction would decrease the hole binding energy, translating into $2 \mathrm{D}$ confinement. In both cases, the reduced offresonant carrier occupation would decrease the $\alpha$-parameter, as we observe in Fig. 3 for the QW and SK QD reference samples.

In conclusion, we have investigated the amplitude-phase coupling in a semiconductor optical amplifier based on SML QDs and quantitatively compared it to devices based on SK QDs and QWs. SML QDs promise to unite the advantages of OD localization with a high DOS. The high density of localization centers leads to an efficient lateral coupling and a very fast gain recovery. The SML QD carrier reservoir induces large phase changes after an optical perturbation. The $\alpha$-parameter exceeds the values typically observed in QDs by an order of magnitude. If lower $\alpha$-parameters are desired for applications, a QW reservoir for the SML QDs analogous to the well-established DWELL structure might be advantageous. Cooling will result in a better confinement of electrons, ${ }^{13}$ and thus a probably reduced $\alpha$-parameter due to less free carrier transitions. While the large amplitude-phase coupling in SML QDs at room temperature places limits on the applicability for linear opto-electronic devices, a large $\alpha$-parameter and the ultrafast gain dynamics of the SML QD based SOA are promising features for nonlinear applications such as cross-phase modulation.

This research was funded by German Research Council (DFG) within the CRC 787 and GRK 1558.

${ }^{1}$ P. Borri, W. Langbein, J. M. Hvam, F. Heinrichsdorff, M.-H. Mao, and D. Bimberg, IEEE Photonics Technol. Lett. 12, 594-596 (2000).

${ }^{2}$ J. Vazquez, H. Nilsson, J. Zhang, and I. Galbraith, IEEE J. Quantum Electron. 42, 986-993 (2006).

${ }^{3}$ A. J. Zilkie, J. Meier, M. Mojahedi, P. J. Poole, P. Barrios, D. Poitras, T. J. Rotter, C. Yang, A. Stintz, K. J. Malloy, P. W. E. Smith, L. Fellow, J. S. Aitchison, P. W. E. Smith, and J. S. Aitchison, IEEE J. Quantum Electron. 43, 982-991 (2007).

${ }^{4}$ H. Schmeckebier, C. Meuer, D. Bimberg, C. Schmidt-Langhorst, A. Galperin, and C. Schubert, Semicond. Sci. Technol. 26, 014009 (2011).

${ }^{5}$ I. L. Krestnikov, M. Straßburg, M. Caesar, A. Hoffmann, U. W. Pohl, D. Bimberg, N. N. Ledentsov, P. S. Kopév, Z. I. Alferov, D. Litvinov, A. Rosenauer, and D. Gerthsen, Phys. Rev. B 60, 8695 (1999).

${ }^{6}$ I. L. Krestnikov, N. N. Ledentsov, A. Hoffmann, and D. Bimberg, Phys. Status Solidi A 183, 207-233 (2001).

${ }^{7}$ A. Lenz, H. Eisele, J. Becker, J.-H. Schulze, T. D. Germann, F. Luckert, K. Potschke, E. Lenz, L. Ivanova, A. Strittmatter, D. Bimberg, U. W. Pohl, and M. Dahne, J. Vac. Sci. Technol., B 29, 04D104 (2011).

${ }^{8}$ S. Sengupta, A. Mandal, H. Ghadi, S. Chakrabarti, and K. L. Mathur, J. Vac. Sci. Technol., B 31, 03 C136 (2013).

${ }^{9}$ D. Arsenijević, C. Liu, A. Payusov, M. Stubenrauch, and D. Bimberg, IEEE Photonics Technol. Lett. 24, 906-908 (2012).

${ }^{10}$ T. D. Germann, A. Strittmatter, U. W. Pohl, D. Bimberg, J. Rautiainen, M. Guina, and O. G. Okhotnikov, J. Cryst. Growth 310, 5182 (2008).

${ }^{11}$ B. Herzog, N. Owschimikow, J.-H. Schulze, R. Rosales, Y. Kaptan, M. Kolarczik, T. Switaiski, A. Strittmatter, D. Bimberg, U. W. Pohl, and U. Woggon, Appl. Phys. Lett. 107, 201102 (2015).

${ }^{12}$ B. Lingnau, K. Lüdge, B. Herzog, M. Kolarczik, Y. Kaptan, U. Woggon, and N. Owschimikow, Phys. Rev. B 94, 014305 (2016).

${ }^{13}$ S. Harrison, M. P. Young, P. D. Hodgson, R. J. Young, M. Hayne, L. Danos, A. Schliwa, A. Strittmatter, A. Lenz, H. Eisele, U. W. Pohl, and D. Bimberg, Phys. Rev. B 93, 085302 (2016).

${ }^{14}$ M. W. Fleming and A. Mooradian, Appl. Phys. Lett. 38, 511 (1981).

${ }^{15}$ C. H. Henry, IEEE J. Quantum Electron. 18, 259-264 (1982).

${ }^{16}$ A. V. Uskov, E. P. O'Reilly, D. McPeake, N. N. Ledentsov, D. Bimberg, and G. Huyet, Appl. Phys. Lett. 84, 272-274 (2004). 
${ }^{17}$ B. Lingnau, W. W. Chow, and K. Lüdge, Opt. Express 22, 4867 (2014).

${ }^{18}$ C. Harder, K. Vahala, and A. Yariv, Appl. Phys. Lett. 42, 328-330 (1983).

${ }^{19}$ W. Rideout, B. Yu, J. LaCourse, P. K. York, K. J. Beernink, and J. J. Coleman, Appl. Phys. Lett. 56, 706-708 (1990).

${ }^{20}$ G. P. Agrawal and C. M. Bowden, IEEE Photonics Technol. Lett. 5, 640-642 (1993).

${ }^{21}$ G. Liu, X. Jin, and S. L. Chuang, IEEE Photonics Technol. Lett. 13, 430-432 (2001).

${ }^{22}$ Z. C. Xu, D. Birkedal, M. Juhl, and J. M. Hvam, Appl. Phys. Lett. 85, 3259 (2004).

${ }^{23}$ M. van der Poel, E. Gehrig, O. Hess, D. Birkedal, and J. M. Hvam, IEEE J. Quantum Electron. 41, 1115 (2005).

${ }^{24}$ J. Muszalski, J. Houlihan, G. Huyet, and B. Corbett, Electron. Lett. 40, 428 (2004).

${ }^{25}$ T. Newell, D. Bossert, A. Stintz, B. Fuchs, K. Malloy, and L. Lester, IEEE Photonics Technol. Lett. 11, 1527-1529 (1999).
${ }^{26}$ I. O’Driscoll, T. Piwonski, C.-F. Schleussner, J. Houlihan, G. Huyet, and R. J. Manning, Appl. Phys. Lett. 91, 071111 (2007).

${ }^{27} \mathrm{~K}$. Pötschke, "Investigation of the formation of quantum dots in the Stranski-Krastanow and submonolayer growth mode," Ph.D. thesis (Technical University Berlin, 2009).

${ }^{28}$ D. Bimberg and U. W. Pohl, Mater. Today 14, 388-397 (2011).

${ }^{29}$ M. Kolarczik, N. Owschimikow, B. Herzog, F. Buchholz, Y. I. Kaptan, and U. Woggon, Phys. Rev. B 91, 235310 (2015).

${ }^{30} \mathrm{~K}$. Lüdge, Nonlinear Laser Dynamics: From Quantum Dots to Cryptography, edited by K. Lüdge (Wiley-VCH, Weinheim, 2012).

${ }^{31}$ S. Schneider, P. Borri, W. Langbein, U. Woggon, R. Sellin, D. Ouyang, and D. Bimberg, IEEE J. Quantum Electron. 40, 1423-1429 (2004).

${ }^{32}$ S. Melnik, G. Huyet, and A. V. Uskov, Opt. Express 14, 2950-2955 (2006).

${ }^{33}$ F. Grillot, B. Dagens, J.-G. Provost, H. Su, and L. F. Lester, IEEE J. Quantum Electron. 44, 946-951 (2008).

${ }^{34}$ N. Tessler and G. Eistenstein, IEEE J. Quantum Electron. 29, 1586-1595 (1993). 